\title{
Effects of IFN- $\gamma$ coding plasmid supplementation in the immune response and protection elicited by Trypanosoma cruzi attenuated parasites
}

Cecilia Pérez Brandán ${ }^{1 *}$ (D) Andrea C. Mesías ${ }^{1}$, Cecilia Parodi ${ }^{1}$, Rubén O. Cimino², Carolina Pérez Brandán³, Patricio Diosque and Miguel Ángel Basombrío

\begin{abstract}
Background: Previous studies showed that a naturally attenuated strain from Trypanosoma cruzi triggers an immune response mainly related to a Th2-type profile. Albeit this, a strong protection against virulent challenge was obtained after priming mice with this attenuated strain. However, this protection is not enough to completely clear parasites from the host. In T. cruzi infection, early Interferon-gamma (IFN- $\gamma$ ) is critical to lead type 1 responses able to control intracellular parasites. Therefore we evaluated whether the co-administration of a plasmid encoding murine IFN- $\gamma$ could modify the immune response induced by infection with attenuated parasites and improve protection against further infections.

Methods: C57BL/6J mice were infected intraperitoneally with three doses of live attenuated parasites in combination with plasmid pVXVR-mIFN- $\gamma$. Before each infection dose, sera samples were collected for parasite specific antibodies determination and cytokine quantification. To evaluate the recall response to $T$. cruzi, mice were challenged with virulent parasites 30 days after the last dose and parasite load in peripheral blood and heart was evaluated.

Results: As determined by ELISA, significantly increase in T. cruzi specific antibodies response was detected in the group in which pVXVR-mIFN- $\gamma$ was incorporated, with a higher predominance of lgG2a subtype in comparison to the group of mice only inoculated with attenuated parasites. At our limit of detection, serum levels of IFN- $\gamma$ were not detected, however a slight decrease in IL-10 concentrations was observed in groups in which pVXVR-mIFN- $\gamma$ was supplemented. To analyze if the administration of pVXVR-mIFN- $\gamma$ has any beneficial effect in protection against subsequent infections, all experimental groups were submitted to a lethal challenge with virulent bloodstream trypomastigotes. Similar levels of challenge parasites were detected in peripheral blood and heart of mice primed with attenuated parasites alone or combined with plasmid DNA. Expansion of IgG antibodies was not significant in TCC+ pVXVR-mIFN- $\gamma$; however, the overall tendency to sustain a Th2 profile was maintained.

Conclusions: Overall, these results suggest that administration of plasmid pVXVR-mIFN- $\gamma$ could have beneficial effects on host specific antibody production in response to T. cruzi attenuated infection; however, this outcome is not reflected in an improved protection against further virulent infections.
\end{abstract}

Keywords: Trypanosoma cruzi, IFN-, , Attenuated infection

\footnotetext{
* Correspondence: cecilia.perezbrandan@conicet.gov.ar

${ }^{1}$ Instituto de Patología Experimental, Consejo Nacional de Investigaciones

Científicas y Técnicas (CONICET), Universidad Nacional de Salta, Salta-Capital,

Argentina

Full list of author information is available at the end of the article
} 


\section{Background}

Trypanosoma cruzi is a kinetoplastid parasite capable of infecting mammalian hosts leading in humans to the development of a number of clinical manifestations known as Chagas disease. This endemic disorder is of great importance in Central and South America since several million people are infected [1]. However, in recent years the increasing percentage of infected people in nonendemic areas due to migration influx has been a major focus of attention [2]. Chagas disease presents broad immunopathological profiles, ranging from asympthomatic cases; single digestive forms with megaesophagus and megacolon; single cardiac forms with intense myocarditis; or digestive and cardiac forms appearing together $[3,4]$. Several years after infection, $30-40 \%$ of the infected persons develop progressive irreversible tissue damage. Currently, chemotherapy for Chagas disease patients is limited to the administration of benznidazole or nifurtimox. These drugs are highly effective in the acute phase, in congenital cases and in children with chronic infection, however; recent studies indicate a limitation in the efficacy of these drugs in chronic adults [5]. In most of the cases, people receiving drug therapy interrupt the treatment as a result of the severity of the side effects associated to nitro compounds [6]. Additionally, restriction of people living in endemic areas to the closer health centers deprives them from receiving a prompt diagnosis and the adequate treatment [7]. For many parasitic diseases, vaccines had the potential to overcome these misfortunes. Several immunoprophylactic as well as immunotherapeutic attempts have been made in order to prevent $T$. cruzi establishment and persistence in the host [8-12]. Still, to date, there is no vaccine licensed for Chagas disease, neither for humans nor for veterinary use.

In $T$. cruzi infection, the innate and adaptive immune responses play a crucial role in parasite control. These responses involve macrophages, natural killer (NK) cells, $\mathrm{T}$ and $\mathrm{B}$ lymphocytes, and the production of proinflammatory Th1 cytokines such as Interferon gamma (IFN- $\gamma$ ), Tumor Necrosis Factor Alpha (TNF- $\alpha$ ) and interleukin 12 (IL-12) [13]. Also, the recognition of pathogen-associated molecular patterns (PAMP) by Toll Like receptors (TLRs), guides for activation of $\mathrm{B}$ and $\mathrm{T}$ cells, highlighting the important role of TLRs in connecting innate and acquired immunity [14]. IFN- $\gamma$ is a key cytokine, secreted by NK cells and other cell types upon IL-12 generation, which has been shown to be crucial in guiding the development of naïve $\mathrm{CD} 4^{+} \mathrm{T}$ cells towards a Th1 phenotype as well as activating macrophages for the production of nitric oxide which is responsible for parasite clearance [15-17]. $\mathrm{CD}^{+} \mathrm{T}$ cells also secrete IFN- $\gamma$ and their importance on parasite control has been well documented [18-20]. Still, it was recently shown that Th17 cells, another subset of CD4 ${ }^{+}$ $\mathrm{T}$ cells, confer significantly stronger protection against T. cruzi than even Th1 cells [21]. In any event, the role of IFN- $\gamma$ in controlling intracellular parasite infections is crucial. Despite the response orchestrated, T. cruzi can induce IL-10 production by dendritic cells and persist indefinitely in the host $[22,23]$.

It has been described that the naturally attenuated TCC strain from T. cruzi triggers a Th2-type immune profile in contraposition to virulent strains which are mainly related to a Th1-type one [24]. Albeit the Th2type outline triggered by TCC infection, this attenuated strain has been extensively used by our group in prime/ boost/challenge experiments [25-31]. This evaluation was also extended to genetically modified mutant clones from this same strain [32, 33]. Even though protection against further infections is strong enough to control parasite replication, is not sufficient to completely clear the parasites from the host. With this in mind we wondered if the addition of an external source of IFN- $\gamma$ could change or improve the immunological and protective effect of attenuated TCC parasites. In this work, we describe the enhancement in the specific antibody responses of mice after infection with TCC attenuated parasites co-administered with plasmid DNA encoding murine IFN- $\gamma$. However, this upgrade in the particular humoral response elicited did not correlate with a significant reduction in parasite burden after challenge with virulent parasites.

\section{Methods}

\section{Parasites and plasmid preparation}

For the infection assays, metacyclic trypomastigotes from the naturally attenuated TCC strain from $T$. cruzi were used [34]. Briefly, epimastigotes were grown at $28^{\circ}$ $\mathrm{C}$ in liver digested neutralized tryptose medium (LDNT), supplemented with $10 \%$ fetal bovine serum (FBS) until they reached log-phase. Metacyclics trypomastigotes were then obtained by epimastigotes differentiation under chemically defined conditions (TAU 3AAG medium). Complement resistant forms were purified using normal non decomplemented serum, quantified in a Neubauer chamber and further used to inoculate experimental animals. Virulent blood trypomastigotes forms from the Tulahuen strain were prepared by routinely biweekly passage through $\mathrm{BALB} / \mathrm{c}$ mice. The eukaryotic expression plasmid pVXVR-mIFN- $\gamma$ was gently donated by Dr. Gomez Hernandez from Brazil. Concisely, mIFN- $\gamma$ was amplified from splenocytes cDNA previously stimulated with lipopolysaccharides (LPS) and then cloned in pVXVR plasmid, a fusion between plasmids pCDNA3 and VR1012. Final construction, pVXVR-mIFN- $\gamma$ was transformed into Escherichia coli $\mathrm{DH} 5 \alpha$ competent cells, grown in LB media containing 
$100 \mu \mathrm{g} / \mathrm{ml}$ ampicillin, and purified by anion-exchange chromatography using the Endo-free Qiagen maxiprep kit (Qiagen) as per manufacturer recommendations and then quantified using Nanodrop 2000 (Thermo Scientific).

\section{Infection in mice}

Six to 8 week old male C57BL/6 J mice were used throughout these studies. Parasites and plasmid DNA were administered intraperitoneally (IP). For this, $10^{5}$ purified metacyclic trypomastigotes/mouse from the TCC strain were used and/or $50 \mu \mathrm{g} /$ mouse of plasmid pVXVR-mIFN- $\gamma$ according to the particular experiment. Three doses were delivered at 4-week intervals. Control groups consisting of empty plasmid and non-infected animals were included in the experiment.

\section{T. cruzi-specific IgGs ELISA}

During the infection period with attenuated parasites and plasmid DNA, serum samples were collected and aliquots were maintained at $-80{ }^{\circ} \mathrm{C}$ until use for IgGs determination. Total immunoglobulin $\mathrm{G}$ antibodies (1:2500 dilution-SIGMA-ALDRICH) against T. cruzi were measured by the enzyme-linked immunosorbent assay (ELISA) using T. cruzi epimastigote homogenate as antigen $(1 \mu \mathrm{g} / 100 \mu \mathrm{l}$-Coating Buffer/well). To identify the antibody subtypes, plates were coated as above, blocked with Phosphate Buffer Solution (PBS) 5\% non-fat dry milk, and then incubated with serum samples $(1: 100$ dilution, $100-\mu \mathrm{l} /$ well) for $1 \mathrm{~h}$, biotin-conjugated goat anti-mouse Ig subtypes (IgG1 or IgG2a-1:3500 dilutionBD Pharmigen) for $1 \mathrm{~h}$, and streptavidin-horseradish peroxidase conjugate (BD-Pharmigen) for $1 \mathrm{~h}$ at $37^{\circ} \mathrm{C}$. Color was developed with TMB Substrate Reagent Set (BD-Pharmigen) and monitored at $450 \mathrm{~nm}$ using a Tecan Infinite Pro microplate reader.

\section{Spleen cells culture supernatant}

Spleens were removed from euthanized infected mice, macerated on a sterile mesh, and cells were resuspended in RPMI 1640 medium. Following centrifugation at $160 \times \mathrm{g}$ for $10 \mathrm{~min}$ at $4{ }^{\circ} \mathrm{C}$, the cells were resuspended in a lysis solution $\left(0.17 \mathrm{M}\right.$ Tris $\left.\mathrm{pH} 7.2,0.16 \mathrm{M} \mathrm{NH}_{4} \mathrm{Cl}\right)$ to remove erythrocytes. The remaining splenocytes were washed three times with RPMI and resuspended in RPMI supplemented with $20 \mathrm{mM}$ glutamine, $10 \%$ $\mathrm{NaHCO}_{3}$ and $10 \%$ fetal bovine serum. Viability of cells was assessed by Trypan blue exclusion and cell number was determined in a Neubauer chamber. Splenocytes $\left(10^{6}\right.$ cells/well in triplicate) were cultured for $48 \mathrm{~h}$ at $37^{\circ}$ $\mathrm{C}$ and $5 \% \mathrm{CO}_{2}$, with or without stimulation with $25 \mu \mathrm{g} /$ $\mathrm{mL}$ of $T$. cruzi epimastigote homogenate or with $50 \mu \mathrm{g} /$ $\mathrm{mL}$ of Phytohaemagglutinin (PHA). Cell culture medium was then collected and aliquots stored at $-80{ }^{\circ} \mathrm{C}$ until use for cytokines determination.

\section{Cytokine response}

Sera samples as well as splenocytes culture supernatant were used for cytokines' measurement (IL-10, TNF- $\alpha$ and IFN- $\gamma$ ) using optEIA enzyme-linked immunosorbent assay (ELISA) kits (BD-Pharmingen), according to the manufacturer's specifications.

\section{Survival rate and parasitemia}

To evaluate the recall response to $T$. cruzi, mice were challenged with $T$. cruzi Tulahuen strain (500 blood trypomastigotes/mouse, IP). Biweekly, blood $(10 \mu \mathrm{l})$ was drawn from the tail tip of mice under slight anesthesia, and the number of parasites per 100 fields (parasitemia) was recorded from fresh blood mounts under microscope $(\times 400)$. Mortality was checked daily until 30 days post challenge and expressed as percentage of survival.

\section{Tissue parasite burden}

Thirty days post virulent challenge mice were sacrificed by carbon dioxide $\left(\mathrm{CO}_{2}\right)$ overexposure. At this point serum samples were taken for determining IgGs expansion and cytokines responses. Heart samples were taken for parasite DNA quantification. Total DNA from heart tissue $(50 \mathrm{mg}$ ) was isolated using the ADN-Puriprep Highway nucleic acid kit (InbioHighway), according to instructions provided by the manufacturer. Total DNA (10 ng) was used as a template, and real-time PCR performed on a LineGene 9640 Sequence Detection System (BIOER) with SYBR Green Supermix (Biosystems) and Tc18SrDNA-specific oligonucleotides (SAT_F 5'GCAG TCGGCKGATCGTTTTCG-3' and SAT_R 5'TTCAG RGTTGTTTGGTGTCCAGTG-3'). Data were normalized to murine TNF- $\alpha$ (TNF_F 5 '-TCCCTCTCATCA GTTCTATGGCCCA-3' and TNF_R 5'CAGCAAGCATCTATGCACTTAGACCCC-3').

\section{Statistical analysis}

Continuous variables, such as antibody levels and parasite concentrations in blood samples, were analyzed with the two-tailed Wilcoxon signed-rank test for time course plots and with the Mann-Whitney or Kruskal-Wallis test for single-day measurements with Dunn's Multiple Comparison Test. Values are expressed as mean with standard errors of the mean (SEM) from at least three independent experiments. Differences between two groups were considered significant and are shown by $" p \quad 0.05,{ }^{* *} p \quad 0.01$, $\%$ *** 0.001 . 


\section{Results}

IFN- $\gamma$ production alters the specific immune response elicited by attenuated parasites

To determine the levels of T. cruzi-specific IgGs after infection with attenuated parasites, serum samples were collected 30 days after each dose. As determined by ELISA, parasite specific antibodies levels increased after each boost and were significantly elevated in the group in which pVXVR-mIFN- $\gamma$ was co-administered in combination with attenuated parasites when compared to the TCC infected group (Fig. 1a, ${ }^{*} p<0.05$ ). To indirectly evaluate the impact of pVXVR-mIFN- $\gamma$ administration on the effector cytokine pattern during infection with attenuated parasites, the levels of parasite specific IgG subtypes were determined by ELISA. IgG1 antibodies were primarily detected in TCC-infected animals with significantly low predominance of IgG2a (Fig. 1b); however when pVXVR-mIFN- $\gamma$ plasmid was administered, a balance between IgG1 and IgG2a was observed with a significantly increase in anti-T. cruzi IgG2a antibodies when compared to TCC-infected animals (Fig. 1b, ${ }^{*} p<$ 0.05 , TCC vs TCC+ pVXVR-mIFN- $\gamma$ ). Because the switch towards a different subtype could be promoted by various cytokines, serum concentrations of regulatory (IL-10) and pro-inflammatory (IFN- $\gamma$ ) cytokines were measured. We observed that sustained boosts with TCC parasites combined with plasmid DNA resulted in increased production of IL-10. However, when pVXVRmIFN- $\gamma$ is added, the concentration of IL-10 at different time points tended to be slightly lower (Fig. 1c, ${ }^{*} p<$ 0.05). IFN- $\gamma$ levels were found below the detection limit of the technique in all of the tested samples. Spleen cells from TCC and TCC + pVXVR-mIFN- $\gamma$ infected animals were also analyzed. Cells were stimulated for $48 \mathrm{hs}$ with T. cruzi antigen and the levels of IFN- $\gamma$ and IL-10 were determined in culture supernatant (Fig. 1d). As in serum samples, IFN- $\gamma$ was not detected at significant levels. IL10 production by splenocytes was similar in TCC and in TCC + pVXVR-mIFN- $\gamma$ infected animals but different from pVXVR-mIFN- $\gamma$ administered animals or naïve ones. Interesting to note is that naïve animals exhibited a lower level of IL-10 compared to animals receiving pVXVR-mIFN- $\gamma$ possibly indicating that the presence of the IFN- $\gamma$ plasmid could be rendering for a lower production of IL-10 by spleen cells. From these results is evident to conclude that the addition of pVXVR-mIFN- $\gamma$

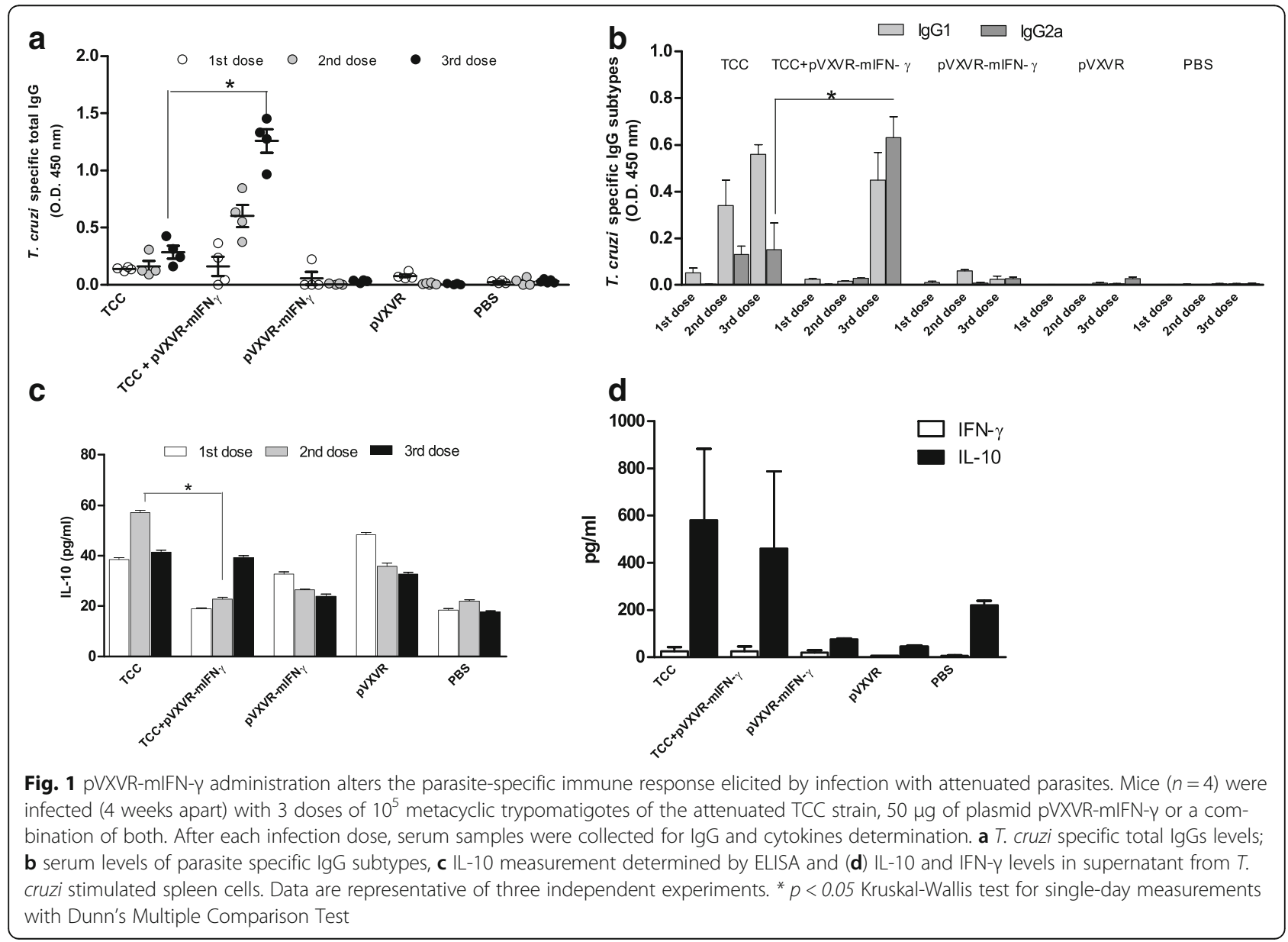


may favor the expansion of the specific IgG response mounted by the prime infection with TCC attenuated parasites. Even more, a tendency in altering the IgG subtype levels (increased in IgG2a profile) and IL-10 production was observed after plasmid addition, indicating that it would be possible to redirect the Th2-type phenotype obtained by the infection with TCC attenuated parasites towards a mixed Th1/Th2 profile.

\section{TCC $+p$ VXVR-mIFN- $\gamma$ elicited protection against in vivo} lethal challenge and parasite replication in heart

To analyze if the immune response elicited by the administration of plasmid pVXVR-mIFN- $\gamma$ in conjunction with live attenuated parasites was protective, all experimental groups were submitted to a lethal challenge with virulent bloodstream trypomastigotes. Animals were infected 30 days after the last dose with attenuated parasites alone or in combination with plasmid pVXVR-mIFN- $\gamma$ and parasites level recorded every week during the acute phase. Survival was monitored daily and all mice from control groups succumbed to death after day 30 post challenge (Fig. 2a). Meanwhile, TCC and TCC + pVXVR-mIFN- $\gamma$ groups showed $100 \%$ survival until the end of follow-up. The level of circulating parasites in peripheral blood from TCC or TCC + pVXVR-mIFN- $\gamma$ infected animals was scarcely detectable (Fig. 2b). In this model and with this technique, the advantage of administrating pVXVR-mIFN- $\gamma$ or not was not evident since no statistically significant differences were found between both groups. However, parasitemia from these groups was significantly different from the control groups (Fig. $2 \mathrm{~b}, * * 0.01$ ). Real time PCR outcome verified the above mentioned results, since a significant reduction in parasite-specific Tc18SrDNA signal in heart of TCC and TCC + pVXVR-mIFN- $\gamma$ infected animals was detected when compared to control groups (Fig. 2c, $* p<0.05$ in TCC and TCC+ pVXVRmIFN- $\gamma$ vs pVXVR-mIFN- $\gamma$ ). Also with this technique the advantages of introducing pVXVR-mIFN- $\gamma$ were not evident since no significant differences with TCC primed animals were detected.

\section{Parasite specific immune response is expanded in reply to $T$. cruzi virulent infection}

In order to evaluate the antibody response elicited in primed animals after challenge with virulent parasites, serum samples were collected 30 days post infection. TCC infected animals exhibited a significant expansion of parasite specific IgG in response to $T$. cruzi virulent infection (Fig. 3a, $" p<0.05$ ). However, in the case in which pVXVR-mIFN- $\gamma$ was co-administered together with attenuated parasites, expansion was significantly diminished. Although not significant, the IgG1 response in TCC + pVXVR-mIFN- $\gamma$ primed/challenged animals was lower than in TCC primed/challenged group. IgG2a was to some extent expanded in the case in which pVXVR-mIFN- $\gamma$ was added, and diminished in TCC-primed mice (Fig. 3b). These data suggested that TCC-primed elicited a parasite-specific IgG response upon challenge infection and that addition of plasmid pVXVR-mIFN- $\gamma$, even not contributing to this expansion, may favor the guidance towards a Th1 phenotype. Serum concentrations of IL-10, TNF- $\alpha$ and IFN- $\gamma$ cytokines were also measured after virulent challenge. The levels of IFN- $\gamma$ and TNF- $\alpha$ were similar in TCC or TCC + pVXVRIFN- $\gamma$ primed/challenged animals in comparison to non-primed animals (Fig. 4a-c). However, unchanged values for IL-10 production were detected among all experimental groups (Fig. 4b).
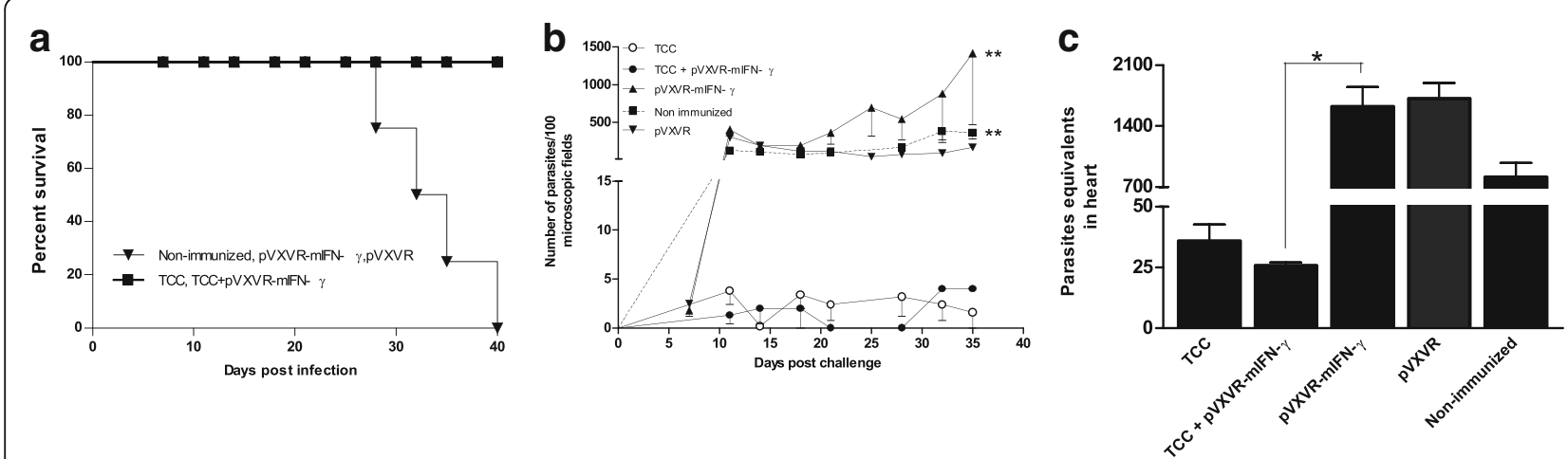

Fig. 2 Control of parasite replication in animals primed with TCC+ pVXVR-mIFN- $\gamma$ after lethal challenge. Thirty days after the last dose with attenuated parasites and plasmid DNA, animals were challenged with 500 blood trypomastigotes from the Tulahuen strain $(n=4)$. a Survival rate during the challenge period; $\mathbf{b}$ parasite load in peripheral blood as determined by fresh blood mount and (c) parasite load in heart as determined by Real Time PCR 30 days post challenge. Data are representative of three independent experiments. * $p<0.05$ and ** $p<0.01$ Kruskal-Wallis test for single-day measurements with Dunn's Multiple Comparison Test 

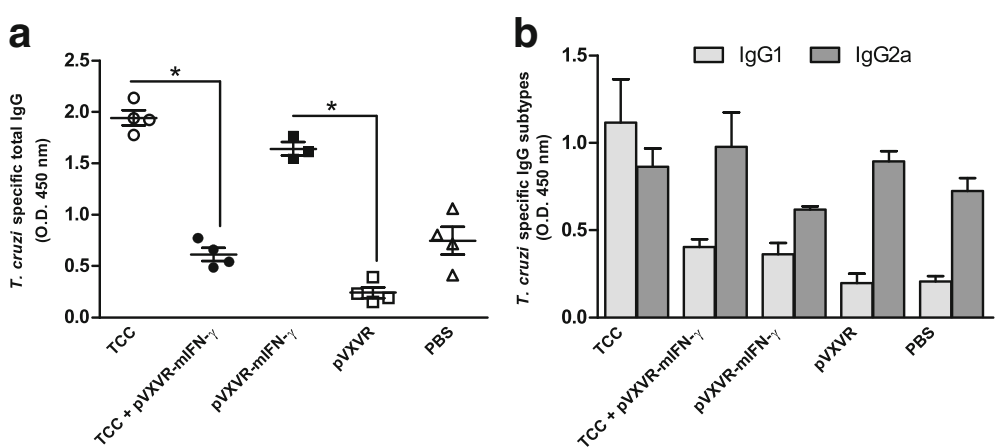

Fig. 3 Expansion of immune specific antibodies in response to T. cruzi infection. Thirty days after challenge serum samples from animals primed with TCC, TCC+ pVXVR-IFN- $\gamma$, pVXVR-IFN- $\gamma$ and PBS were collected for (a) parasite specific IgG antibodies and (b) IgG subtypes by ELISA. Data are representative of three independent experiments. ${ }^{*} p<0.05$ Kruskal-Wallis test for single-day measurements with Dunn's Multiple

Comparison Test

\section{Discussion}

It is known that during the course of many infections, initial IFN- $\gamma$ production is fundamental and may be important in the development of resistance to many intracellular infections such as the one produced by T. cruzi. Also, the activation of the specific cells responsible for the production of this cytokine requires the presence of live parasites [35]. In an attempt to elucidate if parasite specific immune response could be modified or improved, we decided to co-administer, together with highly attenuated parasites, a eukaryotic expression plasmid encoding the murine sequence of the cytokine IFN$\gamma$. Our principal observation is that the addition of the plasmid containing IFN- $\gamma$ significantly improves the generation of parasite specific antibodies in response to prime infection. It is well known that early secreted IFN- $\gamma$ by NK cells activates plasma B cells to produce antibodies in order to lyse extracellular trypomastigotes or to facilitate the opsonization process. Therefore the effect of early introducing a source of IFN $-\gamma$ fostering the natural generation of parasite specific antibodies upon infection is beneficial as demonstrated by our results. The importance of IFN- $\gamma$ in the generation of a
Th1 response and in the formation of IFN- $\gamma$-dependent IgG2a antibodies has been demonstrated since parasite specific antibody response of infected IFN- $\gamma-\mathrm{KO}$ mice was restricted to IgG1 antibodies, being parasite specific IgG2a antibodies absent [36]. TCC-primed animals presented a larger predominance of IgG1 rather than IgG2a antibodies; however, with IFN- $\gamma$ plasmid administration, this phenotype could be slightly biased towards a Th1/ Th2 balance with reduced values of IgG1a and enlarged levels of IgG2a. This switch of Ig-isotype to cytophilic and complement-fixing IgG2a antibodies has also been demonstrated to be induced by IFN- $\gamma$ [37]. Even more, these same results were observed in other infections assays following the same experimental design, in which attenuated parasites and plasmid DNA were administered oral or intramuscularly, indicating that the administration route, especially for plasmid DNA, does not modify the results herein presented (Additional file 1: Figure S1).

When a host has been immunized, either by infection or vaccination, one of the most important parameters to be measured is its capacity to respond against a subsequent infection. All immunized animals were protected
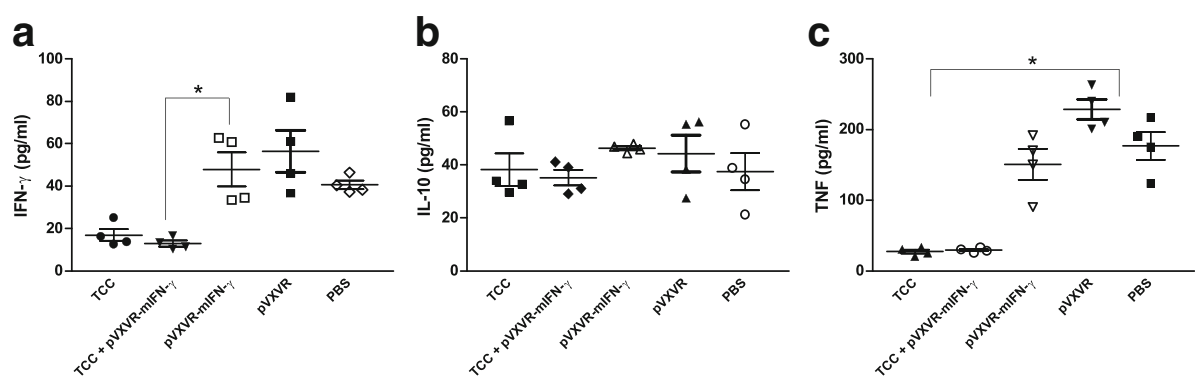

Fig. 4 Cytokines levels in response to virulent T. cruzi infection. Cytokine concentration in serum of TCC and TCC+ pVXVR-IFN-ץ primed animals

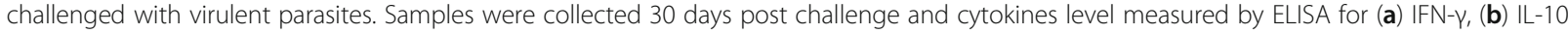
and (c) TNF-a. Data are representative of three independent experiments. ${ }^{*} p<0.05$ Kruskal-Wallis test for single-day measurements with Dunn's Multiple Comparison Test 
against death, with $100 \%$ of these mice surviving the lethal challenge. Virulent infection of primed animals with a lethal dose of bloodstream trypomastigotes led to significantly decreased parasitemias and parasite load in heart samples in all the groups infected with attenuated parasites, regardless administration or not of plasmid coding for IFN- $\gamma$. Interesting to note is that previous additions of pVXVR-mIFN- $\gamma$ solely and later infection with virulent parasites resulted, in some extent, in exacerbated parasite load in blood as well as in heart samples, suggesting that unregulated IFN- $\gamma$ inner production could be unfavorable for the host. These results are supported by the observation that parasite load in mice inoculated with naked plasmid and later infected with virulent parasites resulted in values similar or lower than the control group inoculated with PBS and challenged later (data not shown). All together, these results indicate that infection with attenuated parasites per se is efficient in regulating parasite burden in target organs and peripheral blood and, that the combined administration of pVXVR-mIFN- $\gamma$ has no significant role in helping controlling parasite replication at this time point.

Challenged infection resulted in a rapid and potent expansion of parasite specific antibody response in TCCinfected animals; however this expansion is not favored by pVXVR-mIFN- $\gamma$ addition. One possible explanation for this observation maybe that upregulation of FcR by IFN- $\gamma$ activated macrophages [38] is augmented by an additional source of IFN- $\gamma$, increasing the captured of opsonized parasites, therefore requiring the availability of a larger amount of parasite specific antibodies. Nevertheless, a considerable level of $T$. cruzi specific antibodies was detected in the group of animals primed with pVXVR-mIFN- $\gamma$ plasmid alone, indicating that mice which have never seen $T$. cruzi antigen before but were stimulated with plasmid carrying IFN- $\gamma$ posses the capacity to better respond, at least at the humoral level, to a subsequent virulent infection. This last result is also supported by the fact that animals primed with empty plasmid and later challenged with virulent parasites presented antibodies levels similar to control group inoculated with PBS. The predominance of a Th1 phenotype in expanded specific IgGs is maintained by pVXVRmIFN- $\gamma$ supply during infection with attenuated parasites, as well as after challenge infection. Since it was reported that IgG2a antibodies efficiently mediates clearance of blood trypomastigotes [39], the increase in anti-T. cruzi IgG2a subtype antibodies after challenge of primed animal could be important to reduce parasite proliferation. Additionally, the assessment of the cytokines profile also showed differences among groups. At 30 days post challenge, the levels of IFN- $\gamma$ and TNF- $\alpha$ in primed animals were lower in comparison to nonprimed animals (pVXVR and PBS group). This finding was in concordance with the level of circulating parasites in peripheral blood at this time point, suggesting that IFN- $\gamma$ and TNF- $\alpha$ production in TCC and TCC + pVXVR- IFN- $\gamma$ primed-animals was not longer necessary since parasite clearance already occurred. The elevated levels of TNF- $\alpha$ found within the non-primed groups could have a harmful effect in the host, exacerbating the existing inflammatory response. It has been demonstrated that TNF- $\alpha$ increased during early $T$. cruzi infection is associated to the induction of trypanomicide mechanisms and tissue injury [40]. In this regard, we suggest that a modulated amount of IFN- $\gamma$ and TNF- $\alpha$ secreted after a virulent challenge could be related to a controlled response against the antigen, meanwhile, an exacerbation of this response with elevated levels of both cytokines, as we have shown in the control groups could be responsible for inflammation and consequent tissue damage. Interestingly, in our experiments, IL-10 production did not differ among groups. Similar results were observed by others, in which chronically infected/challenged mice only showed IL-10 production after day 7 post challenge, reaching later, to levels similar to those found in non infected/challenged mice. These authors suggest that IL-10 production, as IFN- $\gamma$ production of chronically infected mice depends on intense proinflammatory signals induced by parasites [41].

Besides stimulating B cells, perhaps the most noticeable effect that IFN- $\gamma$ exerts is the enhancement of microbicidal activity by stimulating macrophages to produce Nitric Oxide (NO) and kill intracellular amastigotes. This evidence has recently been confirmed by means of "Macrophage Insensitive to Interferon Gamma" KO mice, since macrophages from these animals were unable to produce $\mathrm{NO}$ and kill intracellular parasites upon challenge and after priming with IFN- $\gamma$ [42]. On the other hand, the impact of IFN- $\gamma$ in the modulation of chemokine expression in vivo has awakened attention [43]. Therefore, it is quite obvious to postulate that other immune mechanisms, beyond the ones herein studied, might had taken place during this immunization process leading to an improved activation and priming of immune specific cells capable of controlling parasite spread.

\section{Conclusions}

In summary we conclude that TCC + pVXVR-mIFN- $\gamma$ infection successfully primed B cells and induced high parasite specific antibody response with a Th1/Th2 phenotype able to protect against a virulent challenge. However, this increase in the specific antibody response, due to pVXVR-mIFN- $\gamma$ addition, is not enough to improve the control of parasite replication and persistence in the host. Basic immunological studies indicate that antibodies solely are not enough to manage $T$. cruzi 
infection and that parasite control relies mostly on a cellular immune response with predominance of activation of $\mathrm{CD}^{+} \mathrm{T}$ cells $[18,19,44]$. In a previous work we showed that $\mathrm{CD} 8^{+} \mathrm{T}$ cells were induced by infection with TCC attenuated parasites [32] thus we supposed that this response could be very likely strengthened by endogenous IFN- $\gamma$ production and expanded after virulent challenge. Licensed vaccines against many pathogens confer protection by stimulating B cells to produce a wide repertoire of pathogen-specific antibodies. Our most striking observation herein presented, that $T$. cruzi-specific humoral responses elicited by attenuated parasites can be optimized and presumably switch towards a Th1/Th2 balance by plasmid encoding IFNY supplementation, addresses the possibility that other immune-boosting strategies, for therapeutic or immuneprophylactic vaccines approaches, should be deeper inquired into. The results herein presented emphasized the fact that a T. cruzi vaccine should be addressed from a multicomponent point of view, properly activating the humoral and cellular responses needed for protection.

\section{Additional file}

Additional file 1: Figure S1. pVXVR-mIFN- $y$ administration alters the parasite-specific immune response elicited by infection with attenuated parasites by the intramuscular and oral route. Mice $(n=4)$ were infected (4 weeks apart) with 3 doses of $10^{5}$ metacyclic trypomatigotes of the attenuated TCC strain, $50 \mu \mathrm{g}$ of plasmid pVXVR-mIFN- $\gamma$ or a combination of both by the intramuscular (A-C) or oral (B-D) route. After each infection dose, serum samples were collected for (A-B) T. cruzi specific total lgGs levels and (C-D) serum levels of parasite specific lgG subtypes. (PDF $48 \mathrm{~kb})$

\section{Abbreviations \\ $\mathrm{CO}_{2}$ : Carbon dioxide; ELISA: Enzyme-linked immunosorbent assay; FBS: Fetal bovine serum; IFN-ץ: Interferon gamma; IL: Interleukin; IP: Intraperitoneally; LDNT: Liver digested neutralized tryptose medium; LPS: Lipopolysaccharides; NK: Natural killer; NO: Nitric Oxide; PAMP: Pathogen-associated molecular patterns; PBS: Phosphate Buffer Solution; PHA: Phytohaemagglutinin; SEM: Standard errors of the mean; TLRs: Toll Like receptors; TNF-a: Tumor Necrosis Factor Alpha}

\section{Acknowledgements}

We are grateful to Maria Celia Mora for animal care and supervision and to Alejandro Uncos, Renato Uncos and Federico Ramos for skillful technical assistance.

\section{Funding}

This work was supported by grants Fundación Florencio Fiorini and PICT 2014-1625 to CPB. The funders had no role in the design of the study and collection, analysis, and interpretation of data and in writing the manuscript.

\section{Availability of data and materials}

All data generated or analyzed during this study are included in this published article.

\section{Authors' contributions}

$\mathrm{CPB}$ and $\mathrm{MAB}$ conceived and designed the experiments. $\mathrm{CPB}, \mathrm{ACM}, \mathrm{CP}, \mathrm{ROC}$ and $\mathrm{CaPB}$ performed the experiments. $\mathrm{CPB}, \mathrm{ACM}$ and $\mathrm{CP}$ analyzed the data. $\mathrm{CPB}, \mathrm{CaPB}$ and PD contributed reagents/materials/analysis tools. CPB wrote the manuscript. All authors revised the manuscript intensively and approved its final version.

\section{Ethics approval}

All animal protocols adhered to the National Institutes of Health (NIH) "Guide for the care and use of laboratory animals" and were approved by the School of Health Sciences and by the Ethical Committee of the National University of Salta, Argentina ( ${ }^{\circ}$ 014-2011).

\section{Consent for publication}

Not applicable.

\section{Competing interests}

The authors declare that they have no competing interests.

\section{Publisher's Note}

Springer Nature remains neutral with regard to jurisdictional claims in published maps and institutional affiliations.

\section{Author details}

${ }^{1}$ Instituto de Patología Experimental, Consejo Nacional de Investigaciones Científicas y Técnicas (CONICET), Universidad Nacional de Salta, Salta-Capital, Argentina. ${ }^{2}$ Instituto de Investigación de Enfermedades Tropicales, Sede Regional Orán. Universidad Nacional de Salta, Salta-Capital, Argentina. ${ }^{3}$ Estación Experimental Agropecuaria Salta, Instituto Nacional de Tecnología Agropecuaria (INTA), Salta-Capital, Argentina.

Received: 23 June 2017 Accepted: 15 November 2017

Published online: 25 November 2017

\section{References}

1. Stanaway JD, Roth G. The burden of Chagas disease: estimates and challenges. Glob Heart. 2015;10(3):139-44.

2. Liu Q, Zhou XN. Preventing the transmission of American trypanosomiasis and its spread into non-endemic countries. Infect Dis Poverty. 2015;4:60.

3. Bilate AM, Cunha-Neto E. Chagas disease cardiomyopathy: current concepts of an old disease. Rev Inst Med Trop Sao Paulo. 2008;50(2):67-74.

4. Coura JR, Borges-Pereira J. Chagas disease: 100 years after its discovery. A systemic review. Acta Trop. 2010;115(1-2):5-13.

5. Pecoul B, Batista C, Stobbaerts E, Ribeiro I, Vilasanjuan R, Gascon J, Pinazo MJ, Moriana S, Gold S, Pereiro A, et al. The BENEFIT trial: where do we go from here? PLoS Negl Trop Dis. 2016;10(2):e0004343.

6. Viotti R, Vigliano C, Lococo B, Alvarez MG, Petti M, Bertocchi G, Armenti A. Side effects of benznidazole as treatment in chronic Chagas disease: fears and realities. Expert Rev Anti-Infect Ther. 2009;7(2):157-63.

7. Pinheiro E, Brum-Soares L, Reis R, Cubides JC. Chagas disease: review of needs, neglect, and obstacles to treatment access in Latin America. Rev Soc Bras Med Trop. 2017;50(3):296-300.

8. Perez Brandan C, Basombrio MA. Genetically attenuated Trypanosoma cruzi parasites as a potential vaccination tool. Bioengineered. 2012;3(4):242-6.

9. Quijano-Hernandez I, Dumonteil E. Advances and challenges towards a vaccine against Chagas disease. Hum Vaccin. 2011;7(11):1184-91.

10. Rodriguez-Morales O, Monteon-Padilla V, Carrillo-Sanchez SC, Rios-Castro M, Martinez-Cruz M, Carabarin-Lima A, Arce-Fonseca M. Experimental vaccines against Chagas disease: a journey through history. J Immunol Res. 2015; 2015:489758

11. Sanchez-Valdez FJ, Perez Brandan C, Ferreira A, Basombrio MA. Genedeleted live-attenuated Trypanosoma cruzi parasites as vaccines to protect against Chagas disease. Expert Rev Vaccines. 2015;14(5):681-97.

12. Vazquez-Chagoyan JC, Gupta S, Garg NJ. Vaccine development against Trypanosoma cruzi and Chagas disease. Adv Parasitol. 2011;75:121-46.

13. Junqueira C, Caetano B, Bartholomeu DC, Melo MB, Ropert C, Rodrigues MM, Gazzinelli RT. The endless race between Trypanosoma cruzi and host immunity: lessons for and beyond Chagas disease. Expert Rev Mol Med. 2010;12:e29

14. Rodrigues MM, Oliveira AC, Bellio M. The immune response to Trypanosoma cruzi: role of toll-like receptors and perspectives for vaccine development. J Parasitol Res. 2012;2012:507874.

15. Antunez MI, Cardoni RL. IL-12 and IFN-gamma production, and NK cell activity, in acute and chronic experimental Trypanosoma cruzi infections. Immunol Lett. 2000;71(2):103-9.

16. Gutierrez FR, Mineo TW, Pavanelli WR, Guedes PM, Silva JS. The effects of nitric oxide on the immune system during Trypanosoma cruzi infection. Mem Inst Oswaldo Cruz. 2009;104(Suppl 1):236-45. 
17. Vespa GN, Cunha FQ, Silva JS. Nitric oxide is involved in control of Trypanosoma cruzi-induced parasitemia and directly kills the parasite in vitro. Infect Immun. 1994;62(11):5177-82.

18. Padilla AM, Bustamante JM, Tarleton RL. CD8 ${ }^{+} \mathrm{T}$ cells in Trypanosoma cruzi infection. Curr Opin Immunol. 2009;21(4):385-90.

19. Tarleton RL. CD8+ T cells in Trypanosoma cruzi infection. Semin Immunopathol. 2015;37(3):233-8.

20. Vasconcelos JR, Dominguez MR, Neves RL, Ersching J, Araujo A, Santos LI, Virgilio FS, Machado AV, Bruna-Romero O, Gazzinelli RT, et al. Adenovirus vector-induced CD8(+) T effector memory cell differentiation and recirculation, but not proliferation, are important for protective immunity against experimental Trypanosoma cruzi infection. Hum Gene Ther. 2014; 25(4):350-63.

21. Cai CW, Blase JR, Zhang X, Eickhoff CS, Hoft DF. Th17 cells are more protective than Th1 cells against the intracellular parasite Trypanosoma cruzi. PLoS Pathog. 2016;12(10):e1005902.

22. Fernandes MC, Andrews NW. Host cell invasion by Trypanosoma cruzi: a unique strategy that promotes persistence. FEMS Microbiol Rev. 2012;36(3): 734-47.

23. Nagajyothi F, Machado FS, Burleigh BA, Jelicks LA, Scherer PE, Mukherjee S, Lisanti MP, Weiss LM, Garg NJ, Tanowitz HB. Mechanisms of Trypanosoma cruzi persistence in Chagas disease. Cell Microbiol. 2012;14(5):634-43.

24. Revelli S, Gomez L, Wietzerbin J, Bottasso O, Basombrio MA. Levels of tumor necrosis factor alpha, gamma interferon, and interleukins 4,6, and 10 as determined in mice infected with virulent or attenuated strains of Trypanosoma cruzi. Parasitol Res. 1999;85(2):147-50.

25. Basombrio MA. Trypanosoma cruzi: partial prevention of the natural infection of guinea pigs with a killed parasite vaccine. Exp Parasitol. 1990; 71(1):1-8.

26. Basombrio MA, Arredes H. Long-term immunological response induced by attenuated Trypanosoma cruzi in mice. J Parasitol. 1987;73(1):236-8.

27. Basombrio MA, Arredes H, Uncos DA, Rossi R, Alvarez E. Field trial of vaccination against American trypanosomiasis (Chagas' disease) in domestic guinea pigs. Am J Trop Med Hyg. 1987;37(1):57-62.

28. Basombrio MA, Arredes HR, Rossi R. Molina de Raspi E: Histopathological and parasitological evidence of immunization of mice against challenge with 17 wild isolates of Trypanosoma cruzi. Int J Parasitol. 1986;16(4):375-80.

29. Basombrio MA, Besuschio S. Trypanosoma cruzi culture used as vaccine to prevent chronic Chagas' disease in mice. Infect Immun. 1982;36(1):351-6.

30. Basombrio MA, Besuschio S, Cossio PM. Side effects of immunization with liver attenuated Trypanosoma cruzi in mice and rabbits. Infect Immun. 1982; 36(1):342-50.

31. Basombrio MA, Segura MA, Mora MC, Gomez L. Field trial of vaccination against American trypanosomiasis (Chagas' disease) in dogs. Am J Trop Med Hyg. 1993;49(1):143-51.

32. Perez Brandan C, Padilla AM, Xu D, Tarleton RL, Basombrio MA. Knockout of the dhfr-ts gene in Trypanosoma cruzi generates attenuated parasites able to confer protection against a virulent challenge. PLoS Negl Trop Dis. 2011; 5(12):e1418

33. Sanchez-Valdez FJ, Perez Brandan C, Ramirez G, Uncos AD, Zago MP, Cimino RO, Cardozo RM, Marco JD, Ferreira A, Basombrio MA. A monoallelic deletion of the TCCRT gene increases the attenuation of a cultured Trypanosoma cruzi strain, protecting against an in vivo virulent challenge. PLoS Negl Trop Dis. 2014;8(2):e2696.

34. Basombrío M, Besuschio S. Trypanosoma cruzi culture used as vaccine to prevent chronic Chagas' disease in mice. Infect Immun. 1982;36(1):351-6.

35. Cardillo F, Voltarelli JC, Reed SG, Silva JS. Regulation of Trypanosoma cruzi infection in mice by gamma interferon and interleukin 10: role of NK cells. Infect Immun. 1996;64(1):128-34.

36. Marinho CR, Nunez-Apaza LN, Martins-Santos R, Bastos KR, Bombeiro AL, Bucci DZ, Sardinha LR, Lima MR, Alvarez JM. IFN-gamma, but not nitric oxide or specific lgG, is essential for the in vivo control of low-virulence Sylvio X10/4 Trypanosoma cruzi parasites. Scand J Immunol. 2007;66(2-3): 297-308.

37. Snapper CM, Paul WE. Interferon-gamma and B cell stimulatory factor-1 reciprocally regulate Ig isotype production. Science. 1987;236(4804):944-7.

38. Umekita LF, Mota I. Role of the mononuclear phagocytic system in the immune and nonspecific clearance of Trypanosoma cruzi bloodstream trypomastigotes. Braz J Med Biol Res. 1989;22(12):1489-95.
39. Brodskyn Cl, Silva AM, Takehara HA, Mota I. IgG subclasses responsible for immune clearance in mice infected with Trypanosoma cruzi. Immunol Cell Biol. 1989;67(Pt 6):343-8.

40. Machado FS, Martins GA, Aliberti JC, Mestriner FL, Cunha FQ, Silva JS. Trypanosoma cruzi-infected cardiomyocytes produce chemokines and cytokines that trigger potent nitric oxide-dependent trypanocidal activity. Circulation. 2000;102(24):3003-8.

41. Marinho CR, Bastos KR, Sardinha LR, Grisotto MG, Lima MR, Alvarez JM. Challenge of Trypanosoma cruzi chronically infected mice with trypomastigotes activates the immune system and reduces subpatent parasitemia levels. J Parasitol. 2004:90(3):516-23.

42. Lykens JE, Terrell CE, Zoller EE, Divanovic S, Trompette A, Karp CL, Aliberti J, Flick MJ, Jordan MB. Mice with a selective impairment of IFN-gamma signaling in macrophage lineage cells demonstrate the critical role of IFNgamma-activated macrophages for the control of protozoan parasitic infections in vivo. J Immunol. 2010;184(2):877-85.

43. Aliberti JC, Souto JT, Marino AP, Lannes-Vieira J, Teixeira MM, Farber J, Gazzinelli RT, Silva JS. Modulation of chemokine production and inflammatory responses in interferon-gamma- and tumor necrosis factor-R1deficient mice during Trypanosoma cruzi infection. Am J Pathol. 2001;158(4): 1433-40.

44. Dos Santos VF, Pontes C, Dominguez MR, Ersching J, Rodrigues MM, Vasconcelos JR. CD8(+) T cell-mediated immunity during Trypanosoma cruzi infection: a path for vaccine development? Mediat Inflamm. 2014;2014: 243786

\section{Submit your next manuscript to BioMed Central and we will help you at every step:}

- We accept pre-submission inquiries

- Our selector tool helps you to find the most relevant journal

- We provide round the clock customer support

- Convenient online submission

- Thorough peer review

- Inclusion in PubMed and all major indexing services

- Maximum visibility for your research

Submit your manuscript at www.biomedcentral.com/submit

) Biomed Central 